Infección de localización quirúrgica en cirugía de colon

\title{
Surgical site infection in colon surgery
}

doi.org/10.23938/ASSN.0374

J.A. Del Moral Luque ${ }^{1,2}$, G. Rodríguez Caravaca ${ }^{1,3}$

\section{Sr. Director:}

En primer lugar, agradecemos al Dr. Balén su carta Infección de localización quirúrgica en cirugía de colon ${ }^{1}$ en relación al artículo Incidencia de infección de localización quirúrgica en cirugía de colon y adecuación de la profilaxis antibiótica: estudio de cohortes prospectivo publicado por nuestro grupo de trabajo en Anales del Sistema Sanitario de Navarra ${ }^{2}$.

Dentro de las infecciones relacionadas con la asistencia sanitaria (IRAS) las infecciones de localización quirúrgica (ILQ) son, junto a las urinarias y respiratorias, las complicaciones más frecuentes a nivel hospitalario. Al menos un tercio de dichas infecciones podría prevenirse mediante distintas estrategias de vigilancia y control eficaces y costo-efectivas ${ }^{3}$.

Cuando se pretende evaluar la incidencia de ILQ lo ideal es la realización de estudios de cohortes prospectivos, puesto que los registros retrospectivos acarrean importantes limitaciones metodológicas, además de una considerable probabilidad de sesgos y errores. El diseño de nuestro trabajo es por tanto de alta evidencia científica y cuenta con una extensa serie de casi 800 intervenciones recogidas durante ocho años, siendo este uno de sus puntos fuertes más destacables.

La infección de la herida quirúrgica es la complicación más habitual entre los pacientes operados por cirugía colorrectal electiva ${ }^{4}$. La prevención de la infección debe iniciarse en la fase preoperatoria y durante el momento de la intervención, lo que implica conocer los factores de riesgo que puedan empeorar el pronóstico del paciente, entre ellos la no adecuación de la profilaxis antibiótica. El uso rutinario de profilaxis en todos los pacientes sometidos a cirugía colorrectal es apoyado por numerosos estudios en la literatura ${ }^{5-7}$.

Nuestro trabajo demuestra una muy alta adecuación de la profilaxis antibiótica en la serie recogida de cirugía de colon, del $91,9 \%$. Consideramos que la profilaxis fue correcta cuando se cumplieron los cinco criterios evaluados (duración, inicio, elección, dosis y vía del antibiótico), tomando
1. Unidad de Medicina Preventiva. Hospital Universitario Fundación Alcorcón. Madrid.

2. Programa Internacional de Doctorado. Universidad Rey Juan Carlos. Madrid.

3. Departamento de Medicina Preventiva y Salud Pública. Universidad Rey Juan Carlos. Madrid.

Recepción: 16/10/2018

Aceptación definitiva: 24/10/2018

\section{Correspondencia:}

Juan Antonio del Moral Luque

Unidad de Medicina Preventiva

Hospital Universitario Fundación Alcorcón

C/ Budapest, 1

28922 Alcorcón (Madrid)

E-mail: j.antoniodelmoral@gmail.com 
como referencia el protocolo hospitalario vigente. Como se refleja en el estudio, en 17 pacientes no se pudo documentar la aplicación de la profilaxis, tras una exhaustiva búsqueda tanto en su ficha específica de recogida de datos como en la historia clínica electrónica, lo que no implica que todos ellos tuvieran una inadecuada profilaxis antibiótica (o ausencia de ella).

Recordemos que el objetivo del trabajo fue evaluar el porcentaje de administración y adecuación al protocolo de la profilaxis antibiótica en la cirugía de colon, así como el efecto de su inadecuación en la incidencia de ILQ. Tal y como apunta el Dr. Balén ${ }^{1}$, hubo variables recogidas y analizadas que no se llegaron a plasmar en el artículo, puesto que no se ceñían estrictamente a los objetivos marcados para esta publicación. Entre ellas, si la intervención quirúrgica se realizó mediante abordaje laparoscópico o por laparotomía. En ese sentido, y como no podía ser de otra manera, de 2008 a 2016 vemos una clara tendencia hacia el empleo de la vía laparoscópica frente a la abierta, aunque en el global del estudio las cirugías laparoscópicas solo representaron el 27,5\%. Como señala el Dr. Balén ${ }^{1}$, el abordaje laparoscópico reduce la infección quirúrgica respecto a la laparotomía ${ }^{8-10}$, siendo la vía abierta un factor de riesgo en la aparición de ILQ. Esto se evidencia tanto en nuestra serie de colon ( $R R=2,5$; IC95\% 1,1-5,7; p=0,03) como en otros recientes trabajos de nuestro grupo para cirugía abdominal ${ }^{11,12}$.

Respecto al criterio "tiempo de inicio" de la profilaxis antibiótica, dicha administración en nuestro hospital está protocolizada para realizarse entre 30 y 60 minutos antes de la incisión. No quedó registrado si cuando existió incumplimiento fue por exceso o por defecto en este criterio temporal. Agradecemos al Dr. Balén ${ }^{1}$ su propuesta en ese sentido, al igual que la novedosa línea de investigación que nos apunta -la utilización o no de preparación mecánica intestinal y/o su asociación con la profilaxis antibiótica vía oral- para la reducción de las tasas de ILQ ${ }^{13}$. A día de hoy la vía de administración utilizada en nuestro centro es exclusivamente intravenosa.
Compartimos con el Dr. Balén ${ }^{1}$ la necesidad de incidir en una mejora futura de los sistemas de registro de todos los posibles factores de riesgo de infección quirúrgica, tanto en la cirugía de colon como en el resto de procedimientos quirúrgicos hospitalarios. Nos referimos no solo a los históricamente establecidos en la literatura, sino también a los recomendados por el Protocolo Infección Quirúrgica Zero en 2017 -como el rasurado, uso de clorhexidina alcohólica al $2 \%$, normotermia y normoglucemia perioperatorias ${ }^{14} \mathrm{e}$ incluso a otras variables como la utilización de oxígeno suplementario y drogas vasoactivas, el empleo de drenajes y transfusiones o la experiencia del cirujano, que ya cuentan con múltiples estudios que avalan su importancia en la prevención de las $\operatorname{ILQ}^{15-17}$ y cuya monitorización y control contribuirían a un incremento de la seguridad clínica y calidad asistencial de nuestros pacientes.

\section{BIBLIOGRAFÍA}

1. BALÉN E. Infección de localización quirúrgica en cirugía de colon. An Sist Sanit Navar 2018; 41: 401-402.

2. Del Moral JA, Alonso M, Gil P, Fernández JM, DurÁN M, RodríguEz G. Incidencia de infección de localización quirúrgica en cirugía de colon y adecuación de la profilaxis antibiótica: estudio de cohortes prospectivo. An Sist Sanit Navar 2017; 40: 371-377.

3. Yokoe DS, Classen D. Improving patient safety through infection control: a new healthcare imperative. Infect Control Hosp Epidemiol 2008; 29: S3-S11.

4. Fraccalvieri D, Kreisler E, Flor B, Torres A, MuÑOZ A, MATEO F et al. Factores predictivos de infección de herida en cirugía colorrectal. Estudio observacional multicéntrico de casos y controles. Cir Esp 2014; 92: 478-484.

5. Nelson RL, Glenny AM, Song F. Antimicrobial prophylaxis for colorectal surgery. Cochrane Database Syst Rev 2009; 1: CD001181.

6. Bratzler DW, Dellinger EP, Olsen KM, Perl TM, Auwaerter PG, Bolon MK et al. Clinical practice guidelines for antimicrobial prophylaxis in surgery. Am J Health Syst Pharm 2013; 70: 195-283.

7. RuIZ J, BADIA JM. Medidas de prevención de la infección del sitio quirúrgico en cirugía ab- 
dominal. Revisión crítica de la evidencia. Cir Esp 2014; 92: 223-231.

8. Romy S, Eisenring MC, Bettschart V, Petignat C, Francioli P, Troillet N. Laparoscope use and surgical site infections in digestive surgery. Ann Surg 2008; 247: 627-632.

9. Balén E, Suárez J, Ariceta I, Oronoz B, Herrera J, LERA JM. Cirugía laparoscópica en las enfermedades colorrectales. An Sist Sanit Navar 2005; 28 (Suppl 3): 67-80.

10. Poon JT, Law WL, Wong IW, Ching PT, Wong LM, FAN JK et al. Impact of laparoscopic colorectal resection on surgical site infection. Ann Surg 2009; 249: 77-81.

11. Del Moral JA, Sánchez T, Gil P, Fernández JM, Hijas AI, Rodríguez G. Efecto de un Plan de Mejora de Calidad y Seguridad Clínica en la incidencia de infección de sitio quirúrgico en apendicectomía. Estudio cuasi-experimental. Cir Cir 2018; 86: 437-445.

12. Colás E, Del Moral JA, Gil P, Fernández JM, Alonso M, VILLAR MC et al. Incidence of surgical site infection and risk factors in rectal surgery: A prospective cohort study. Cir Esp 2018; doi: 10.1016/j.ciresp.2018.06.007.

13. Chen M, Song X, Chen L, Lin Z, Zhang X. Comparing mechanical bowel preparation with both oral and systemic antibiotics versus mechanical bowel preparation and systemic antibiotics alone for the prevention of surgical site infection after elective colorectal surgery: A Meta-analysis of randomized controlled clinical trials. Dis Colon Rectum 2016; 59: 70-78.

14. Sociedad Española de Medicina Preventiva, Salud Pública e Higiene. Protocolo Infección Quirúrgica Zero 2017. Disponible en: http:// infeccionquirurgicazero.es/images/stories/ recursos/protocolo/2017/3-1-17-documentoProtocolo-IQZ.pdf. Consultado el 12 de octubre de 2018.

15. Tang R, Chen HH, Wang YL, Changchien CR, CHEN JS, Hsu KC et al. Risk factors for surgical site infection after elective resection of the colon and rectum: a single-center prospective study of 2,809 consecutive patients. Ann Surg 2001; 234: 181-189.

16. Hoffmann J, Shokoun MH, Damm P, Jensen R. A prospective, controlled study of prophylactic drainage after colonic anastomoses. Dis Colon Rectum 1987; 30: 449-452.

17. Mallol M, Sabaté A, Kreisler E, Dalmau A, CamPRUBI I, TRENTI L et al. Incidencia de la infección de la herida quirúrgica en cirugía colorrectal electiva y su relación con factores perioperatorios. Cir Esp 2012; 90: 376-381. 
